\title{
Effect of interaction of atomic electrons on ionization of an insulator in swift heavy ion tracks
}

\author{
A.A. Baranov ${ }^{\text {a }}$, N.A. Medvedev ${ }^{\text {b }}$, A.E. Volkov ${ }^{\text {c,* }}$, N.S. Scheblanov ${ }^{\mathrm{d}}$ \\ ${ }^{a}$ LAPTH, Universite de Savoie, 9, Chemin de Bellevue, BP 110, 74941 Annecy-le-Vieux, France \\ ${ }^{\mathrm{b}}$ CFEL at DESY, Notkestr. 85, 22607 Hamburg, Germany \\ ${ }^{\mathrm{c}}$ National Research Center Kurchatov Institute, Kurchatov Sq. 1, 123182 Moscow, Russia \\ ${ }^{\mathrm{d}}$ Laboratoire Hubert Curien, UMR CNRS 5516, Université de Lyon, 18 rue Benoît Lauras, Bat. F, 42000 Saint-Etienne, France
}

\section{A R T I C L E I N F O}

\section{Article history:}

Received 10 August 2011

Received in revised form 24 November 2011

Available online 14 January 2012

\section{Keywords:}

Multiionization

Swift heavy ion

Track

Electronic excitations

Monte-Carlo

binary collisions approximation

statistical energy deposition

\begin{abstract}
A B S T R A C T
Effects of applications of two different models of atomic ionizations in MC simulations describing excitation of the electronic subsystem of an insulator in tracks of swift heavy ions decelerated in the electronic stopping regime are investigated. The first mechanism is based on binary collisions of a fast projectile with atomic electrons considered as independent. The second one assumes redistribution of the energy transferred to an atom from an ion between the atomic electrons before autoionization of the excited atom. It is demonstrated that an appreciable difference occurs only in the kinetics of the most energetic electrons appeared during the ionization. This difference affects only a small fraction of the excess electronic energy in the far periphery of a track.
\end{abstract}

(c) 2012 Elsevier B.V. All rights reserved.

\section{Introduction}

The largest part of the energy loss of swift heavy ions (SHI) with energies higher than $1 \mathrm{MeV} / \mathrm{amu}$ and masses more than 20 proton mass is realized due to excitations of the electronic subsystem of a target. The electronic stopping $S_{e}(3-10 \mathrm{keV} / \mathrm{nm})$ constitutes more than $95 \%$ of the energy loss of such an ion. Conversion of even a little part of the excess electronic energy into excitations of the target lattice can stimulate structure and phase transformations in the nanometer vicinities of the SHI trajectories [1].

Recently, the original Monte-Carlo (MC) model simulating the initial electronic kinetic in SHI tracks was successfully applied to a quantitative description of X-ray spectroscopy experiments investigating luminescence from the ionized track cores in $\mathrm{SiO}_{2}$ [2-4]. This MC model was based on the Asymptotic Trajectory Monte-Carlo (ATMC) method. The binary collisions approximation (BCA) was applied in [2-4] to describe ionization events as results of binary interactions of a projectile as well as of generated fast electrons with atomic electrons. No interactions and/or correlations between atomic electrons were assumed during ionization of a target atom.

\footnotetext{
* Corresponding author. Tel.: +7 49919691 78; fax: +7 4991961612 .

E-mail address: a.e.volkov@list.ru (A.E. Volkov).
}

Such interactions were taken into account in models describing multiple atomic ionizations resulting from binary collisions of slow charged particles with atoms, e.g. the statistical energy deposition model (the SED-model) [5-7]. Later the SED was expanded [8] for calculations of multiple ionization cross sections of atoms and molecules by fast ions [9-11] as well as for calculations of the spectra of electrons appearing in SHI tracks in water $[12,13]$.

It was demonstrated recently [14] that interactions between target electrons during ionizations result only in about of $2 \%$ changes in the predicted values of the electronic energy loss $S_{e}$ of a SHI. However, different models of atomic ionizations predict different spectra of generated fast electrons that may result at least in differences of the spatial distributions of the deposited excess energy affecting the structure transformations in ion tracks.

In this paper we use Monte-Carlo simulations (see [2-4] for details) in order to compare the effects of the electronic spectra predicted by the BCA and the SED models of ionization of target atoms by a swift heavy ion on the subsequent kinetics of the electronic subsystem of a wide band gap dielectric (silica) in a SHI track.

\section{Monte-Carlo simulations}

For the both models of atomic ionization we used the ATMC model where swift heavy projectiles are considered as point-like 
particles moving with a constant velocity $V_{p}$ along the classical trajectories. ATMC is the reasonable assumption for ions with energies higher than $1 \mathrm{MeV} / \mathrm{amu}$. The trajectories of SHIs are assumed as straight lines because the elastic energy losses $\left(S_{n}\right)$ resulting from elastic collisions of such projectiles with target atoms are negligible in comparison to $S_{e}\left(S_{n} \ll S_{e}\right)$. The last assumption does not make critical limitations because curvatures of the trajectories can be easily incorporated into the MC model. Detailed description of the physical and numerical background of this MC model can be found in [2-4].

This kind of semi-classical Monte Carlo method is a standard tool of modeling particle transport in solids (see e.g. books [15-18], papers [19-24] and references therein). It assumes that a particle is interacting with a media via a sequence of binary encounters. In Refs. [19,22-24] limits of applicability of such methods were extended by some improvements of the cross-sections of particle scattering which include collective effects (such as plasmon or phonon excitation). Another significant improvement of the classical binary collision theory was made in Refs. $[25,26]$ by including a suitable effective potential. These advanced methods are a subject for a future work and are not included in the present paper where the standard semi-classical MC method is utilized [2-4,15-18,20,21].

Atomic energy levels are used for description of the initial state of the target electrons instead of the band structure of material. The band structure affects the dynamics of the slowest electrons and influences only negligible to high energy electrons which, as it will be demonstrated below, are resulting in the effects discussed in the paper.

To compare the SED and the BCA models of atomic ionizations, solid silica $\left(\mathrm{SiO}_{2}\right)$ was chosen as the target. It has a density of $\rho=2.32 \mathrm{~g} / \mathrm{cm}^{2}$ corresponding to the atomic concentration of $n=6.9 \times 10^{22} \mathrm{~cm}^{-3}$. The target is considered as a homogeneous atom arrangement with fixed positions of atoms because the characteristic times of ionization and initial relaxation of electronic excitations in SHI tracks $\left(10^{-14} \mathrm{~s}\right)$ are much smaller than the atomic oscillation time $\left(\sim 10^{-13} \mathrm{~s}\right)$.

Calcium ions ( $\mathrm{Ca}, M_{\text {ion }}=40 m_{p}, Z_{\text {ion }}=20$ ) with the energy 11.4 MeV/amu similar to the energies provided by the UNILAC linear accelerator (GSI, Darmstadt) were chosen as a projectiles in our simulations. The Barkas formula [27] gives the equilibrium charge $Z_{p}=18.74$ of such an ion in silica.

The simulations were performed for a target layer of $10 \mathrm{~nm}$ thicknesses. The periodical boundary conditions along the $z$-axis coinciding with the projectile trajectory were applied.

\section{Description of the BCA and the SED models of atomic ionizations by a SHI}

\subsection{Binary collisions approximation of atomic ionizations by a SHI}

The BCA model assumes independent instant ionizations when atomic electrons have no time for mutual interactions which could result in redistribution of the energy they got from a SHI. This model assumes that the energies transferred to atomic electrons due to their Coulomb interactions with a projectile are determined by the instant positions of these electrons in a target atom taking into account the randomly chosen impact parameters of a projectile to the atomic nuclei. These positions of electrons are extracted from the relevant electronic wave functions [3]. The emission angles of electrons are unambiguously determined by the transferred energies.

\subsection{Multi-ionization approximations of atomic ionizations by a SHI}

The SED-model assumes multiple ionization of an atom by a charged particle as a two-stage process [7]. At the first stage, a part of the kinetic energy of an ion is transferred to a target atom resulting in excitation of the atomic electronic shells. At the second stage, after the collision, the energy deposited into the atom is statistically redistributed among all atomic electrons resulting finally in auto-ionizations of the excited atom that gives the number and the spectra of appeared free electrons. It is assumed that these auto-ionizations are too slow comparing to the time of an ion-atom collision that allows their description as relaxations of an isolated atom.

The extension of the SED model to swift ions [8] is based on the idea to equate the energy deposited into an atom with the electronic energy loss of a projectile, while the elastic energy losses are neglected. The dependence of the energy transferred by a swift ion to a target atom $T(b)$ on the impact parameter $b$ forms the external input parameter of the SED-model.

The SED model assumes that the probability of $n$-electron ionization of an atom at the given transferred energy $T(b)$ is proportional to the number of final states which can be occupied by $n$ free electrons [7]:

$P_{n}(T)=\frac{C_{n}^{N}(g)^{n} S_{n}\left(E_{k i n} / I_{1}\right)}{\sum_{i=1}^{N} C_{i}^{N}(g)^{i} S_{i}\left(E_{k i n} / I_{1}\right)}$

Here, $C_{i}^{N}$ is the binomial coefficient, $N$ is the total number of electrons in the atom. The function $S_{n}(x)=2^{((n-1) / 2)} \pi^{(n / 2)} x^{(3 n-2) / 2} /$ $(3 n-2)$ !! ( with $\left.\mathrm{x}=E_{\mathrm{Kin}} / I_{1}\right)$ represents the density of final states with $n$ electrons in the continuum. The double factorial $(3 n-2) !$ ! is defined as $(3 n-2) ! !=(3 n-2)(3 n-4)(3 n-6) \ldots$ ending with 2 for even $n$ and with 1 for odd $n$. $E_{\text {Kin }}$ is the total kinetic energy of the ejected $n$ electrons

$E_{k i n}=T(b)-\sum_{i=1}^{n} I_{i}$

and $I_{i}$ is the $i$-th ionization potential of the target atom. The fitting parameter, $g$, corresponds to the averaged matrix element of the ionization of $i$-electrons. In our calculations we took the parameter $g$ equal to 0.001 [10-12], providing the closest coincidence of the electron spectra got from the SED model with the BCA predictions.

The probability density function $F_{n}(E)$ for a free electron to have the kinetic energy in the interval $(E, E+d E)$ when $n$ electrons with the total kinetic energy $E_{k i n}$ are emitted from an atom [7,12]:

$F_{n}(E)=\frac{S_{n-1}\left(E_{k i n}-E\right) \cdot S_{1}(E)}{S_{n}\left(E_{k i n}\right)}$

In the numerical algorithm the energy of the first electron $\left(E_{1}\right)$ emitted from an atom is determined according to Eq. (3). For the next emitted electron the conditional probability distribution is used with the total available energy $\left(E_{k i n}-E_{1}\right)$ :

$F_{n}\left(E / E_{1}\right)=\frac{S_{n-2}\left(E_{k i n}-E_{1}-E\right) \cdot S_{1}(E)}{S_{n}\left(E_{k i n}-E_{1}\right)}$.

This procedure is repeated for $n-1$ electrons and the kinetic energy of the last electron is $E_{n}=E_{k i n}-E_{1}-\ldots-E_{n-1}$ providing the energy conservation [13].

Eqs. (1-4) give the spectra of free electrons generated due to target ionizations. Unfortunately, the SED model cannot trace from which atomic shell an electron was excited, thus, cannot predict the distributions of holes at different shells of an ionized atom.

No preferred directions for ejection of electrons can be selected within the SED-model. In [12] three different models of the angular distributions of emitted electrons were proposed: (1) the isotropic emission; (2) the angular emission corresponding to binary collisions of the ion with free electrons; (3) the uniform angular distribution of emitted electrons in the cone with the angle of $120^{\circ}$. Ref. [12] demonstrated that the difference between these initial angular distributions does not affect the electronic kinetics already at a 
distance larger than $1 \mathrm{~nm}$ from the ion trajectory. In this paper we choose the uniform momentum distribution of the emitted electrons in the cone of $120^{\circ}$.

We used two models in order to calculate the energy $T(b)$ transferred by a SHI to a target atom which is the input parameter for the SED-model:

(1) The Unitary Convolution Approximation model (UCA) by Schiwietz et al. (CasP 4.0-code) [28,29] which gives the dependence of the energy transferred to an atom on the impact parameter.

(2) The Local Density Approximation model (LDA) proposed by Lindhard and Scharff [30], which describes the electronic energy loss of SHI as those for an ion moving in a gas of free electrons. This model is well applicable when the parameter $Z_{p} / v$ ( $v$ is the ion velocity) measured in the atomic units is much smaller than unity [11]. But it was also successfully applied for projectiles having $Z_{p} / v$ of the order of unity as well [10]. For Ca ion with the energy $11.4 \mathrm{MeV} / \mathrm{amu}$ and the charge $Z_{p}=18.74$ this ratio is equal to 0.87 .

In the LDA model, when a projectile moves along a straight line trajectory, the energy transferred into the electronic subsystem of an atom is determined by:

$T(\boldsymbol{b})=\frac{4 \pi Z_{p}^{2} e^{4}}{m_{e} v^{2}} \int_{-\infty}^{+\infty} d z \rho(\boldsymbol{r}) L[\rho(\boldsymbol{r}), v]$,

where $\rho(\boldsymbol{r})$ is the electronic density in the target atom which can be taken e.g. from the CasP-code $[28,29]$ (isolated atoms); the $z$-axis is chosen along the projectile trajectory $(\mathbf{r}=\{\mathbf{b}, z\}) . L[\rho(\boldsymbol{r}), v]$ is the stopping power, which in the case of the electron gas can be approximated by [31]:

$$
\begin{aligned}
L[\rho(\boldsymbol{r}), v] & =C(\chi)\left(\frac{v}{v_{F}(\boldsymbol{r})}\right)^{3}, v \leqslant v_{F} ; L[\rho(\boldsymbol{r}), v] \\
& =\ln \left(\frac{2 v^{2}}{\omega_{p}(\boldsymbol{r})}\right)-\frac{3}{5}\left(\frac{v}{v_{F}(\boldsymbol{r})}\right)^{2}, v \geqslant v_{F} .
\end{aligned}
$$

In Eq. (5) $v_{F}(\boldsymbol{r})=\left(3 \pi^{2} \rho(\boldsymbol{r})\right)^{1 / 3}(h / 2 \pi m)$ is the Fermi velocity, $h$ is the Plank constant; $\omega_{p}(\boldsymbol{r})=\sqrt{4 \pi e^{2} \rho(\boldsymbol{r}) / m}$ is the plasma frequency; and $C(\chi)=\frac{1}{2\left(1-\chi^{2} / 3\right)^{2}}\left[\ln \left(\frac{1+2 \chi^{2} / 3}{\chi^{2}}\right)-\frac{1-\chi^{2} / 3}{1+2 \chi^{2} / 3}\right]$ with $\chi=e^{2} /$ $4 \pi v_{F}(r)$.

Fig. 1 demonstrates the dependencies of the energy transferred to the atomic electronic system $T(b)$ on the impact parameter $b$ calculated in the framework of LDA and CasP models for collisions of a Ca ion (11.4 MeV/amu) with oxygen O (a) and silicon Si (b) atoms.

The transferred energy required for ionization of an atom must be higher than the first ionization potential $I_{1}$. Therefore, taking into account that $T(b)$ is the monotonic function (see Fig. 1 ) the

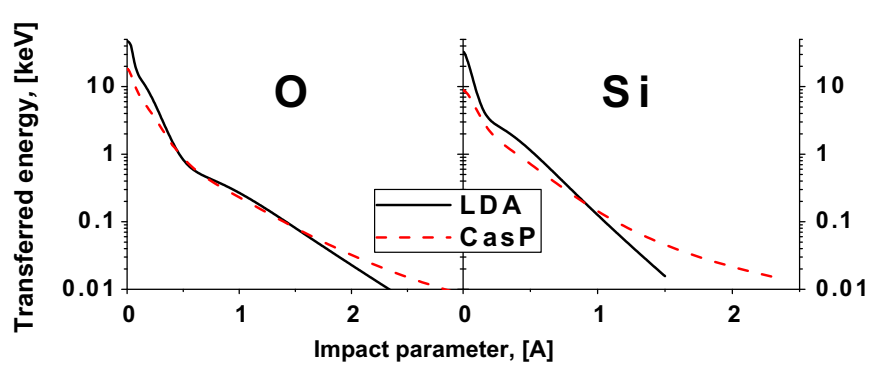

Fig. 1. The dependence of the energy $T(b)$ transferred to the atomic electrons on the impact parameter $b$ in collisions of a calcium ion (11.4 MeV/amu) with oxygen $\mathrm{O}$ and silicon $\mathrm{Si}$ atoms. maximum impact parameter $b_{\max }$ which is necessary for ionization of an atom is determined by the condition:

$T\left(b_{\max }\right)=I_{1}$.

We assume that the occasional realizations of the values of the impact parameter $b$ are uniformly distributed within the circle area restricted by the radius $b_{\max }$ determined by Eq. (7). In this case the realized value of $b$ can be linked in the MC algorithm with the random value $\gamma_{1}$ uniformly distributed in the interval $(0,1][18]$ :

$b=b_{\max } \sqrt{\gamma_{1}}$

For the random spatial distribution of scattering centers (atoms), the realized free path of a projectile can be expressed through the random value $\gamma_{2}$ uniformly distributed in the interval $(0,1][2,4]$ :

$l=-<l>\ln \gamma_{2}$

where $\langle l\rangle$ is a mean free path of a SHI scattered at these centers. The value of the mean free path $\langle l\rangle$ was adjusted in order to fit the linear energy loss of a fast projectile to the SRIM data $\left(S_{e}=d E\right.$ / $d x=2.67 \mathrm{keV} / \mathrm{nm}$ for $11 \mathrm{MeV} / \mathrm{amu} \mathrm{Ca}$ ion in silica) [32].

\section{Monte-Carlo simulations of free electron transport}

In order to investigate how the difference in the initial electronic spectra reveals in the electronic transport and the ionizations produced by generated free electrons, we apply the same ATMC algorithm as in Refs. [2-4]. The free paths of an electron related to different scattering channels are described by equations similar to Eq. (9) with the mean free paths defined by the crosssections of ionizations or elastic electron scattering on atoms related to these channels.

In contrast to a SHI, elastic collisions of free electrons with target atoms are included in the scattering model because they change significantly the directions of motion of electrons resulting in diffusive rather than ballistic behavior of spatial propagation of the excess energy of the electronic ensemble in a SHI track. The Mott's cross-section was used to calculate the electronic mean free paths for elastic scattering of electrons $[4,17,19]$. This cross-section depends on the energy of an electron and on the atomic number of a colliding atom.

Only single ionizations produced by fast electrons are taken into account because multiple ionizations of an atom have negligible probabilities for an electron impact [3]. The Gryzinski's ionization cross-section was used to describe inelastic collisions between free and atomic electrons (see $[2-4,17,19]$ and references therein). This inelastic cross-section depends only on the energy of a traced electron and the ionization potential of a bound electron to be ionized. Inelastic processes are taken into account only when the energy of a traced electron exceeds the ionization potential of a bound electron. The shell, which an electron is being ionized from, is chosen randomly according to the relative cross-sections of ionization of different shells of an atom [3]. The energy transferred in the traced electron-electron collision determines the scattering angle. The polar angle was stochastically chosen from the interval $[0,2 \pi)$. Spreading and collisions of the subsequent generations of free electrons are described in the same manner.

Averaging over the statistics accumulated during the code iterations gives the spatial and temporal distributions of the densities of free electrons and holes at different atomic shells (the last is only for the BCA model of ionization of target atoms by a projectile) as well as the energy distributions of these electronic excitations in the nanometric vicinity of the projectile trajectory. 


\section{Results and discussions}

The electronic energy loss $S_{e}$ of an ion is used as the input parameter in the discussed ionization models. Therefore, first of all, in order to compare the results of MC simulations, the electronic energy losses $S_{e}$ of Ca ion were fitted [2-4] to the SRIM2008 code [32] value $\left(S_{e}=d E / d x=2.67 \mathrm{keV} / \mathrm{nm}\right.$ ) for the SED (with the both inputs from CasP 4.0 and from LDA) as well as for the BCA ionization models.

\subsection{The energy spectrum of primary electrons}

Fig. 2 presents the energy distributions of primary free electrons appeared due to ionizations of target atoms by a projectile and contained at distances up to $b_{\max }$ from the ion trajectory. This figure demonstrates that for both realizations (CasP and LDA) of the SED model, the obtained spectra of fast electrons differ significantly from those predicted by the BCA model for the high energy region. This occurs despite of the fitting of the parameter $g$ of the SED model to the value providing the best coincidence of the SED electronic spectra with those calculated in the framework of the BCA model. The high energy "tails" of these spectra do not coincide.

The SED model gives systematically a lower number of the energetic electrons $\left(E_{e}>10 \mathrm{keV}\right)$ than that obtained from the BCA model because in the SED model the probability of ionization of a larger number of electrons increases with an increase of the energy transferred to an atom. This results in a distribution of the energy deposited by a SHI to an atom among the larger number of ionized electrons, which, in turn, decreases their individual energies and leads to a lack of high energy electrons. In the BCA model, the energy transferred by a SHI to an atomic electron is restricted only by the mass ratio $\left(m_{e} / M_{i}\right)$, and electrons can obtain the energy up to the maximum value $E_{e}{ }^{\max }=24 \mathrm{keV}$ allowed by the head-on collisions with an $11.4 \mathrm{MeV} / \mathrm{amu}$ ion. Note, that in case of a choice of the parameter $g=1$ (see Section 3.2) the SED spectra would decrease even faster, compared to BCA, giving dramatic difference for the number of high energy electrons.

For the different realizations of the SED model (CasP and LDA inputs), the spectra of ejected electrons differ considerably again in the high energy region. The CasP mechanism predicts the wider distribution of the transferred energy $T(b)$ on the impact parameter than the LDA approach (see Fig. 1). The larger impact parameters result in the smaller energies of the ejected electrons that appears in the sharper decrease of the energy dependence of the electronic spectra predicted by the SED-CasP model in the high energy region.

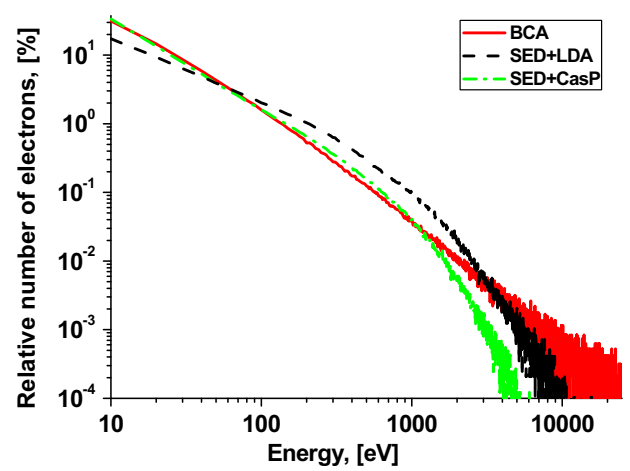

Fig. 2. The energy distributions of primary electrons predicted by the different models of atomic ionization of silica by a swift (11.4 MeV/amu) Ca ion: the BCA model and the SED-model with transferred energy calculated in the framework of LDA (SED + LDA) and Casp 4.0 (SED + CasP) models.

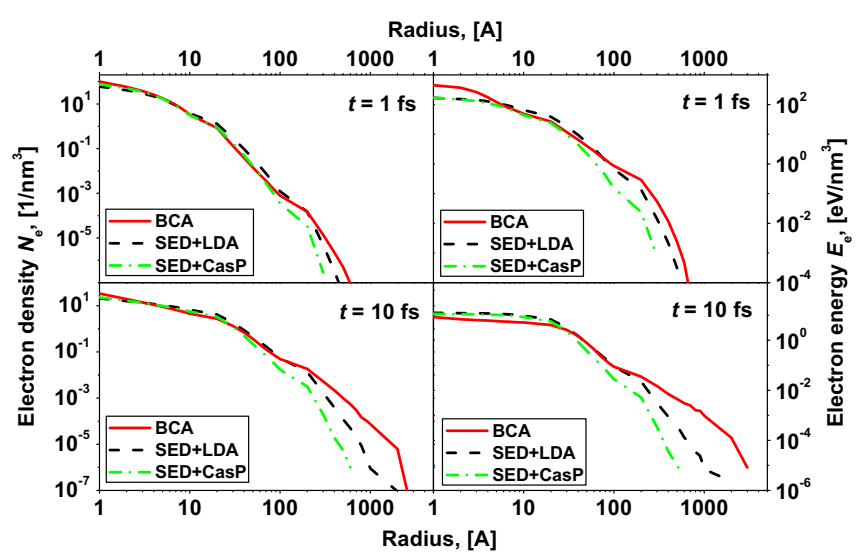

Fig. 3. The radial distributions of (a) the density and (b) the energy density of free electrons predicted by different models of atomic ionization of a track of $\mathrm{Ca}$ ion (11 MeV/amu) in $\mathrm{SiO}_{2}$ at $1 \mathrm{fs}$ and $10 \mathrm{fs}$ after the projectile passage.

Summarizing, the difference between the models of atomic ionization by a SHI appears mostly in the high energy part of the spectrum of primary ionized free electrons generated in tracks.

\subsection{Spatial propagation of electronic excitations}

Fig. 3 demonstrates that the averaged over $10^{4}$ statistical runs radial distributions of the density and the energy density of free electrons calculated in the framework of different ionization models in the $100 \AA$ vicinity of the projectile trajectory are practically the same at 1 as well as at $10 \mathrm{fs}$ after the ion passage. This region contains more than $99 \%$ of the excess energy of the electronic subsystem at these times.

However, a significant difference between the model predictions occurs at long distances from the track axis, where the parameters of these distributions are mostly formed by the faster electrons. As discussed above, the SED models give the smaller number of primary ionized high energy electrons than the BCA model. This results in the wider radial distributions of the densities of free electrons as well as the energy density they accumulated predicted by the BCA model.

In spite of the fact that only about $1 \%$ of the excess energy of the electronic ensemble is contained at the track periphery, this difference might be important for some effects governed by fast electrons, e.g. the incubation fluence effect providing the initial changes of the optical properties of alkali-halides irradiated with SHIs (e.g. bleaching) [33,34] due to impurity neutralization by fast electrons at large distances from the ion trajectories. The spectra of electrons emitted from the surface of irradiated target [35] predicted by different models of atomic ionizations in SHI tracks should also be different. These effects give a chance for experimental verifications of the discussed ionization models.

\section{Conclusions}

In this paper we compared results of applications of the independent particle binary collisions approximation (BCA) and the statistical energy deposition (SED-LDA, SED-CasP) models describing atomic ionizations by swift heavy ions decelerated in a dielectric (silica) in the electronic stopping regime.

On example of silica $\left(\mathrm{SiO}_{2}\right)$ it was demonstrated that the spectra of primary ionized electrons predicted by different models differ considerably only in the high-energy region. The SED-model gives a smaller number of fast primary electrons in comparison to the BCA model. Though, these fast electrons accumulate less than $1 \%$ 
of the energy deposited by a swift heavy ion into the electronic subsystem of a target and are contained at the track periphery ( $>10 \mathrm{~nm}$ from the ion trajectory), the observed differences may be important for effects governed by the fastest electrons, as e.g. modification of the irradiated wide band gap insulators at the initial fluencies (incubation fluence effect), or experimentally detectable spectra of electrons emitted from the surface of an irradiated target.

\section{Acknowledgements}

Financial supports from the Russian Foundation for Basic Researches under Contracts No. 10-08-90024, 11-08-91162, 11-0890424 and 11-08-08135 as well as DFG Grant TR 973/1-1 are acknowledged by A.E. Volkov.

\section{References}

[1] F.F. Komarov, Defect and track formation in solids irradiated by superhighenergy ions, Phys.-Usp. 46 (2003) 1253-1282 [Usp. Fiz. Nauk 12 (2003) 1287].

[2] N.A. Medvedev, A.E. Volkov, Monte-Carlo simulations of electronic excitations in swift heavy ion tracks in $\mathrm{SiO}_{2}$, AIP Conf. Proc. 999 (2008) 238-244.

[3] N.A. Medvedev, A.E. Volkov, N.S. Shcheblanov, B. Rethfeld, Early stage of the electron kinetics in swift heavy ion tracks in dielectrics, Phys. Rev. B 82 (2010) 125425.

[4] N.A. Medvedev, A.E. Volkov, B. Rethfeld, N.S. Shcheblanov, Effect of interatomic Auger processes on relaxation of electronic vacancies at deep levels of highly ionized atoms in swift heavy ion tracks, Nucl. Instr. Meth. B 268 (2010) 2870-2873.

[5] A. Russek, M.T. Thomas, Ionization Produced by Atomic Collisions at kev Energies. II, Phys. Rev. 114 (1959) 1538-1540.

[6] A. Russek, Ionization produced by high-energy atomic collisions, Phys. Rev. 132 (1963) 246-261.

[7] A. Russek, J. Meli, Ionization phenomena in high-energy atomic collisions, Physica 46 (1970) 222-243.

[8] C.L. Cocke, Production of highly charged low-velocity recoil ions by heavy-ion bombardment of rare-gas targets, Phys. Rev. A 20 (1979) 749-758.

[9] N.M. Kabachnik, V.N. Kondratyev, Z. Roller-Lutz, H.O. Lutz, Multiple ionization of atoms and molecules in collisions with fast ions: ion-atom collisions, Phys. Rev. A 56 (1997) 2848-2854.

[10] N.M. Kabachnik, V.N. Kondratyev, Z. Roller-Lutz, H.O. Lutz, Multiple ionization of atoms and molecules in collisions with fast ions. II. Ion-molecule collisions, Phys. Rev. A 57 (1998) 990-996.

[11] Z. Kaliman, N. Orlic, N.M. Kabachnik, H.O. Lutz, Theoretical study of orientation effects in multiple ionization of molecules by fast ion impact, Phys. Rev. A 65 (2001) 012708

[12] C. Champion, Multiple ionization of water by heavy ions: a Monte Carlo approach, Nucl. Instr. Meth. B 205 (2003) 671-676.

[13] C. Champion, A. L'hoir, M.F. Politis, P.D. Fainstein, R.D. Rivarola, A. Chetioui, A Monte Carlo code for the simulation of heavy-ion tracks in water, Radiat. Res. 163 (2005) 222-231.
[14] M.D. Barriga-Carrasco, Proton stopping using a full conserving dielectric function in plasmas at any degeneracy, Phys. Rev. E 82 (2010) 046403.

[15] M.J. Berger, Methods in computational physics, in: B. Adler, S. Fernbach, M. Rotenberg, vol. 1, Academic Press Inc., New York, 1963.

[16] W. Eckstein, Computer Simulations of Ion-Solid Interactions, Springer-Verlag, New York, 1991.

[17] A.F. Akkerman, Modeling of charged particles trajectories in matter [in Russian], Nauka, Moscow, 1991. p. 200.

[18] G.S. Fishman, Monte-Carlo Concepts, Algorithms and Applications, SpringerVerlag, New York, 1996. p. 701.

[19] A. Akkerman, M. Murat, J. Barak, Ion track structure calculations in silicon Spatial and temporal aspects, Nucl. Instr. Meth. Phys. Res. B 269 (2011) 16301633.

[20] M.P.R. Waligorski, R.N. Hamm, R. Katz, The radial distribution of dose around the path of a heavy ion in liquid water, Nucl. Tracks Radiat. Meas. 11 (1986) 309.

[21] B. Gervais, S. Bouffard, Simulation of the primary stage of the interaction of swift heavy ions with condensed matter, Nucl. Instr. Meth. Phys. Res. B 88 (1994) 355-364.

[22] A.F. Akkerman, T. Boutboul, A. Breskin, R. Chechik, A. Gibrekhterman, Y. Lifshitz, Inelastic electron interact ions in the energy range $50 \mathrm{eV}-10 \mathrm{keV}$ in insulators: alkali halides and metal oxides, Phys. Stat. Sol. B 198 (1996) 769.

[23] S. Tanuma, C.J. Powell, D.R. Penn, Calculations of electron inelastic mean free paths. III. Data for 15 inorganic compounds over the 50-2000 eV range, Surf. Interface Anal. 17 (1991) 927.

[24] A. Jablonski, C.J. Powell, Improved algorithm for calculating transport cross sections of electrons with energies from $50 \mathrm{eV}$ to $30 \mathrm{keV}$, Phys. Rev. B 76 (2007) 085123.

[25] P. Sigmund, A. Schinner, Binary stopping theory for swift heavy ions, Eur. Phys. J. D 12 (2000) 425-434.

[26] A. Schinner, P. Sigmund, Impact-parameter-dependent electronic stopping of swift ions: I. Binary theory, Eur. Phys. J. D 56 (2010) 41-50.

[27] H.D. Betz, Charge states and charge-changing cross sections of fast heavy ions penetrating through gaseous and solid media, Rev. Mod. Phys. 44 (1972) 465539.

[28] P.L. Grande, G. Schiwietz, The unitary convolution approximation for heavy ions, Nucl. Instr. Meth. B 195 (2002) 55-63. http://www.helmholtz-berlin.de/ people/gregor-schiwietz/casp_en.html.

[29] P.L. Grande, G. Schiwietz, Convolution approximation for the energy loss, ionization probability and straggling of fast ions, Nucl. Instr. Meth. B 267 (2009) 859-865

[30] J. Lindhard, M. Scharff, Energy loss in matter by fast particles of low charge, Mat. Fys. Medd. Dan. Vid. Selsk. 15 (1953) 27

[31] J. Lindhard, A. Winther, Stopping power of electron gas and equipartition rule, Mat. Fys. Medd. K. Dan. Vidensk. Selsk. 4 (1964) 34.

[32] J.F. Ziegler, The stopping of energetic light ions in elemental matter, J, Appl. Phys/Rev. Appl. Phys. 85 (1999) 1249-1272. http://www.srim.org.

[33] K. Schwartz, A.E. Volkov, M.V. Sorokin, C. Trautmann, K.-O. Voss, R. Neumann, M. Lang, Effect of electronic energy loss and irradiation temperature on colorcenter creation in $\mathrm{LiF}$ and $\mathrm{NaCl}$ crystals irradiated with swift heavy ions, Phys. Rev. B 78 (2008) 024120.

[34] K. Schwartz, C. Trautmann, A.S. El-Said, R. Neumann, M. Toulemonde, W. Knolle, Color-center creation in LiF under irradiation with swift heavy ions: dependence on energy loss and fluence, Phys. Rev. B 70 (2004) 184104.

[35] N. Lineva, C. Kozhuharov, S. Hagmann, M. Kramer, G. Kraft, Low-energy electrons emitted in collisions of heavy ions with solid state targets, Nucl. Instr. Meth. B 267 (2009) 891-895. 